\title{
Self-organization in ${ }^{4} \mathrm{He}$ near the superfluid transition in heat flow and gravity
}

\author{
Syunsuke Yabunaka and Akira Onuki \\ Department of Physics, Kyoto University, Kyoto 606-8502, Japan
}

(Dated: November 1, 2018)

\begin{abstract}
We investigate the nonlinear dynamics of ${ }^{4} \mathrm{He}$ slightly below the superfluid transition by integrating model $\mathrm{F}$ equations in three dimensions. When a superfluid is heated from above under gravity, a vortex tangle and sheetlike phase slips both appear near the bottom plate. Then a self-organized superfluid containing high-density vortices and phase slips grows upward, where high-amplitude second sounds are emitted from the self-organized to the ordinary superfluid region. A phase slip sheet often changes into a vortex aggregate and vice versa. The thermal resistance due to these defects produces a constant temperature gradient equal to the gradient of the pressure-dependent transition temperature $T_{\lambda}(p)$. In this self-organized region, the temperature deviation $T-T_{\lambda}(p)$ consists of a negative constant determined by the heat flux $Q$ and time-dependent fluctuations. Its time-average is calculated to be $155 \mathrm{nK}$ for $Q=11.2 \mathrm{erg} / \mathrm{cm}^{2} \mathrm{~s}$ in good agreement with the experiment (W.A. Moeur et al., Phys. Rev. Lett. 78, 2421 (1997)).

PACS numbers: 67.25.dj, 67.25.dk, 05.70.Jk,05.70.Ln
\end{abstract}

\section{INTRODUCTION}

The critical phenomena in ${ }^{4} \mathrm{He}$ near the superfluid transition have been studied with exceedingly high precision of the temperature $T\left(\sim 10^{-9} \mathrm{deg}\right)^{1-4}$. Such experiments have thus been used to confirm or predict universal relations near the critical point. Moreover, in this system, a number of unique nonequilibrium situations can be found in the nonlinear response regime. In particular, the superfluid transition is very sensitive to applied heat flow $Q^{5,6}$. For example, if $T=T(z)$ is above the transition temperature $T_{\lambda}$ at one end of the cell and below $T_{\lambda}$ at the other end, a HeI-HeII interface emerges separating superfluid and normal fluid regions ${ }^{7-13}$. Here $T$ is nearly constant in the superfluid and has a finite gradient in the normal fluid, so the temperature gradient is almost discontinuous across the interface. In experiments, it emerges when ${ }^{4} \mathrm{He}$ in a normal fluid is cooled from a boundary below $T_{\lambda}$ or when ${ }^{4} \mathrm{He}$ in a superfluid is warmed from a boundary above $T_{\lambda}$. This interface has a thickness proportional to $Q^{-1 / 2}$ at small $Q$ and is thus a unique singular object. Some authors were also interesred in the heat capacity in heat flow near the superfluid transition ${ }^{10}$. Another interesting case is to quench liquid ${ }^{4} \mathrm{He}$ from its normal to its superfluid phase by a mechanical expansion ${ }^{14}-16$. In such phase ordering with a multi-component order parameter, topological singularities (vortices in ${ }^{4} \mathrm{He}$ ) should be proliferated in an early stage after quenching 5,14 .

The superfluid transition can also be greatly influenced by gravity due to the pressure dependence of $T_{\lambda}(p)$. In ${ }^{4}$ He near the transition, the specific heat exhibits a sizable logarithmic singularity, but the isothermal compressibility has almost no singularity ${ }^{1,5}$, resulting in nearly homogeneous density profiles for not large cell length on earth. Thus the height-dependence of $T_{\lambda}(p)$ is the main origin of inhomogeneity in equilibrium ${ }^{4} \mathrm{He}$, preventing precise measurements of the critical phenomena in ${ }^{4} \mathrm{He}^{2,3}$. If the $z$ axis is taken in the upward direc- tion on earth, $T_{\lambda}(p)$ decreases with the height $z$ as

$$
T_{\lambda}(p)=T_{\lambda \mathrm{bot}}(1+G z),
$$

where $T_{\lambda \text { bot }}$ is the transition temperature at the cell bottom and $G=\left|d T_{\lambda} / d p\right| \rho g / T_{\lambda} \cong 0.6 \times 10^{-6} \mathrm{~cm}^{-1}$ at saturated vapor pressure with $g$ being the gravity constant. The origin of the $z$ axis is at the cell bottom. We introduce the local reduced temperature as

$$
\tau \equiv T / T_{\lambda}(p)-1=\left(T / T_{\lambda \mathrm{bot}}-1\right)-G z,
$$

which depends on $z$ and represents the distance from the lambda line in the $p$ - $T$ phase diagram Therefore, equilibrium states are noticeably inhomogeneous in the temperature region

$$
|\tau| \lesssim G h
$$

where $h$ is the vertical cell length. Remarkably, in the temperature range $\tau(h)<0<\tau(0)$ at homogeneous $T$ (in equilibrium), a gravity-induced two-phase coexistence is possible with an upper region in a superfluid and a lower region in a normal fluid ${ }^{17,18}$. The thickness $\xi_{g}$ and the characteristic reduced temperature $\tau_{g}$ of the gravityinduced interface is determined by the balance $\tau=G \xi$, where $\xi=\xi_{0}|\tau|^{-2 / 3}$ is the correlation length with $\xi_{+0}=$ $1.4 \AA$. Hence they are given by

$$
\ell_{g}=\xi_{+0}\left(\xi_{+0} G\right)^{-2 / 5}, \quad \tau_{g}=\left(\xi_{+0} G\right)^{3 / 5},
$$

which are about $10^{-9}$ and $10^{-2} \mathrm{~cm}$ on earth, respectively. It is worth noting that application of an electric field $E$ or a magnetic field $H$ gives rise to a shift of $T_{\lambda}$ by an amount proportional to $E^{2}$ or $H^{2}$. If such a field is inhomogeneous, it leads to a gradient of $T_{\lambda}$, as well as the earth gravity ${ }^{18}$.

Furthermore, both gravity $g$ and heat flux $Q$ can be crucial near the lambda line. Hereafter $Q$ will be measured in units of $\mathrm{erg} / \mathrm{cm}^{2} \mathrm{~s}$. If heated from below, they both serve to decrease $\tau$ with increasing the height and 
the interface changes over from the gravity-induced one to the heat-induced one at $Q \sim 1$ with increasing $Q$. (We may determine this crossover $Q$ by equating the thicknesses of these interfaces.) On the other hand, if heated from above, they can compete to produce new nonequilibrium states. In fact, Moeur et al $\underline{\underline{19}-21}$ realized selforganized superfluid in ${ }^{4} \mathrm{He}$ heated from above, where the temperature gradient and the gradient of $T_{\lambda}(p)$ were balanced as

$$
T_{\lambda} \frac{d \tau}{d z}=\frac{d T}{d z}-T_{\lambda} G=0
$$

yielding a homogeneous $\tau=-\tau_{\text {sos }}(Q)$ with

$$
\tau_{\mathrm{sos}}(Q) \cong 1.0 \times 10^{-8} Q^{0.813}
$$

in the range $5 \lesssim Q \lesssim 60$. This self-organized region extended over a macroscopic region in a $7.4 \mathrm{~mm}$ tall, $2 \mathrm{~cm}$ diameter cylindrical cell. It is surprising that the distance $\tau$ to the lambda line was held fixed at an extremely small negative constant over a macroscopic region. Machta et $a l{ }^{22}$ pointed out that ${ }^{4} \mathrm{He}$ in heat flow is an example of self-organized criticality. However, ${ }^{4} \mathrm{He}$ in heat flow and gravity stays slightly away from the lambda line, so it is not in a "critical state", to be precise.

Notice that the balance relation (5) itself follows from the vortex resistance in the Gorter-Mellink form ${ }^{23}$,

$$
d T / d z=T B(T) Q^{3},
$$

where $B=B_{0}|\tau|^{-\sigma}$ with $B_{0}=5 \times 10^{-29}$ and $\sigma=2.23$ near the transition 24 . The balance $B(T) Q^{3}=G$ then yields $\tau=-\tau_{\text {sos }}^{\mathrm{v}}$ with

$$
\tau_{\mathrm{sos}}^{\mathrm{v}}=\left(B_{0} Q^{3} / G\right)^{1 / \sigma} \cong 1.3 \times 10^{-10} Q^{1.35} .
$$

However, this $\tau_{\text {sos }}^{\mathrm{v}}$ is smaller than the experimental $\tau_{\text {sos }}$ in Eq.(6) by one or two orders of magnitude for $Q \gtrsim 5$. In previous simulations in one dimension 25,26 , another kind of singular objects, phase slips, came into play, producing a thermal resistance in accord with the experiment. In the literature, phase slips were theoretically studied in one dimension $27-29$ and have been observed in quasi-one-dimensional superconductors ${ }^{30}$ and in ${ }^{4} \mathrm{He}$ in narrow apertures 31 . The self-organized superfluid states are analogous to the resistive states in wire superconductors in electric field; however, in our case, phase slips appear as sheets even for macroscopic cell width. This is because the uniaxial effect of gravity is intensified near the lambda line. Similar self-organization should well be realized in ${ }^{4} \mathrm{He}$ in the presence of a gradient of electric or magnetic field, for example, around the tip of a charged needle kept cooler than the surrounding liquid ${ }^{4} \mathrm{He}$.

Originally, the balance relation (5) was predicted for normal liquid ${ }^{4} \mathrm{He}^{32}$. Liquid ${ }^{4} \mathrm{He}$ exhibits strong critical fluctuations of the complex order parameter slightly above the lambda line, which give rise to strong critical enhancement of the thermal conductivity $\lambda^{\underline{1}} \underline{\underline{\underline{n}}}$. For small positive $\tau$, experimental data of $\lambda$ may be fitted to the following power law ${ }^{3}$,

$$
\lambda \cong \lambda_{0} \tau^{-x_{\lambda}} \quad\left(x_{\lambda} \cong 0.45\right),
$$

where $\lambda_{0}$ is a constant. In terms of $\tau$ in Eq.(2), the heat conduction equation reads

$$
C_{p} \frac{\partial \tau}{\partial t}=\frac{\partial}{\partial z} \lambda\left[\frac{\partial \tau}{\partial z}+G\right]
$$

where the specific heat $C_{p}$ is treated to be a constant for simplicity. In steady states, the temperature gradient is $Q / \lambda$ so that

$$
d \tau / d z=\left(Q / T_{\lambda} \lambda_{0}\right) \tau^{x_{\lambda}}-G
$$

If $\tau(h)>0$, the balance in Eq.(4) gives $\tau=\tau_{\text {son }}(Q)$ with

$$
\tau_{\text {son }}(Q)=\left(T_{\lambda} \lambda_{0} G / Q\right)^{1 / x_{\lambda}}=3.8 \times 10^{-9} Q^{-2.22} .
$$

This holds in the region $L-z \gg \ell_{n}(Q)$, where $\ell_{n}(Q)=$ $\tau_{\text {son }}(Q) / G$ is the relaxation length. Weichman and Miller ${ }^{26}$ argued that the balance relation (5) is attained only when the correlation length $\xi=\xi_{0}|\tau|^{-2 / 3}$ is shorter than $\ell_{n}(Q)$. From Eq.(4) this local equilibrium condition is rewritten as $\tau_{\text {son }} \gtrsim \tau_{g} \sim 10^{-9}$. Thus we obtain the upper bound $Q \lesssim 1$ needed for the self-organization above the lambda line. For larger $Q$, the system crosses across the lambda line and a HeI-HeII interface appears. In accord with these results, Moeur et al $\frac{19}{19}$ observed selforganized normal fluid states for $Q \lesssim 1$. Analyzing the nonlinear diffusive equation (10), Weichman and Miller ${ }^{26}$ also predicted a new thermal wave propagating only upward in self-organized normal states. This wave was observed both above and below the lambda line 20,21 .

The physical processes in the self-organized superfluid remain quite unclear, since in the experiments near the transition measured was only the temperature and the simulations were one-dimensional. Realistic threedimensional simulations are needed to investigate how vortices and phase slips come into play. With this purpose, we will present dynamic equations in Sec.II and numerical results in three dimensions in Sec.III.

\section{MODEL EQUATIONS}

We use the renormalized model $\mathrm{F}$ equations ${ }^{4,13}$ accounting for gravity ${ }^{\underline{5}}$. The dynamic variables are the complex order parameter $\psi(\boldsymbol{r}, t)$ and the entropy $s(\boldsymbol{r}, t)$. The superfluid density $\rho_{s}$ and the superfluid momentum density $\boldsymbol{J}_{s}=\rho_{s} \boldsymbol{v}_{s}$ are proportional to $|\psi|^{2}$ and $\operatorname{Im}\left(\psi^{*} \nabla \psi\right)$, respectively, where $\operatorname{Im}(\cdots)$ denotes taking the imaginary part. The Fourier components of $\psi$ and $s$ have wave numbers smaller than a upper cut-off $\Lambda$. The fluctuations with wave numbers larger than $\Lambda$ have already been coarse-grained ${ }^{4,5,13}$. As $\Lambda$ is decreased down to the inverse correlation length $\xi^{-1}$, the renormalization 
effect is suppressed to justify use of the mean-field theory at long wavelengths, where the coefficients of the model are renormalized ones dependent on $\tau$.

In equilibrium superfluid, the average order parameter behaves as $\psi \propto|\tau|^{1 / 3}$. Without gravity, we define the correlation length as $\xi=\xi_{+0} \|\left.\tau\right|^{-2 / 3}$ using $\xi_{+0}=1.4 \AA$. We suppose a reference equilibrium superfluid state, where $\tau=-\tilde{\tau}, \psi=\tilde{\psi}$, and $\xi=\tilde{\xi}$ with

$$
\tilde{\tau}=2.5 \times 10^{-8}, \quad \tilde{\xi}=1.6 \times 10^{-3} \mathrm{~cm} .
$$

Hereafter we set $\Lambda=\tilde{\xi}^{-1}$ and treat the fluctuations with sizes longer than $\tilde{\xi}$. Those with sizes shorter than $\tilde{\xi}$ are little affected by heat flow. The scaled position $\boldsymbol{r} / \tilde{\xi}$, the scaled time $t / \tilde{t}$, the scaled correlation length $\xi / \tilde{\xi}$, and the scaled reduced temperature $\epsilon / \tilde{\epsilon}$ will be written as $\boldsymbol{r}, t, \xi$, and $\epsilon$ in the same notation for simplicity, where

$$
\tilde{t} \cong 0.5 \times 10^{-3} \mathrm{~s}
$$

is the thermal relaxation time on the scale of $\tilde{\xi}$. We introduce the scaled reduced temperatures,

$$
\begin{aligned}
& A(\boldsymbol{r}, t)=\left(T / T_{\lambda b o t}-1\right) / \tilde{\tau}, \\
& \varepsilon(\boldsymbol{r}, t)=\tau / \tilde{\tau}=A-\tilde{G} z,
\end{aligned}
$$

where $\tilde{G}=G \tilde{\xi} / \tilde{\tau}=0.04$ on earth. The equations for the scaled variables $\Psi(\boldsymbol{r}, t)=\psi / \tilde{\psi}$ and $A(\boldsymbol{r}, t) \operatorname{read}^{5}$

$$
\begin{aligned}
\frac{\partial}{\partial t} \Psi & =i \frac{A}{a} \Psi-L^{*}\left[\varepsilon \xi^{-1 / 2}-\nabla^{2}+\xi^{-1}|\Psi|^{2}\right] \Psi, \\
\frac{\partial}{\partial t} M & =a \operatorname{Im}\left(\Psi^{*} \nabla^{2} \Psi\right)+\nabla \cdot \lambda^{*} \nabla A,
\end{aligned}
$$

where $M$ is the scaled entropy deviation defined by

$$
M=A-a^{2} \xi^{-1 / 2}|\Psi|^{2} / 2
$$

and $a$ is a universal number of order unity. The second term $\left(\propto|\Psi|^{2}\right)$ represents a decrease of the entropy in the ordered phase. We set $a=1$ in our simulation. In gravity the correlation length does not exceeds $\ell_{g}$ in Eq.(4), so we define the local correlation length as

$$
\xi=\tilde{\ell}_{g} \tanh \left(1 / \tilde{\ell}_{g}|\varepsilon|^{2 / 3}\right),
$$

where $\tilde{\ell}_{g}=\ell_{g} / \tilde{\xi}=1 / \tilde{G}^{2 / 5}=3.62$. Then we obtain two cases of $\xi \cong|\varepsilon|^{-2 / 3}<\ell_{g}$ and $\xi \cong \ell_{g}<|\varepsilon|^{-2 / 3}$. The scaled kinetic coefficients are written as 4.13

$$
L^{*}=b_{\psi} \xi^{0.325}, \quad \lambda^{*}=b_{\lambda} \xi^{0.675} .
$$

We set $b_{\psi}=0.2$ and $b_{\lambda}=1$ in our simulation ${ }^{5}$.

For ${ }^{4} \mathrm{He}$ near the lambda line, the complete hydrodynamic equations including the dynamic equation for $\psi$ were presented by the Russian group many years ago $\underline{33}$. In the model $\mathrm{F}^{\underline{4}}$, we do not treat the hydrodynamic equation for the mass density $\rho$ and the total momentum density $\boldsymbol{J}=\rho_{n} \boldsymbol{v}_{n}+\boldsymbol{J}_{s}$ neglecting the fluctuations of $\rho$ and $\boldsymbol{J}$,
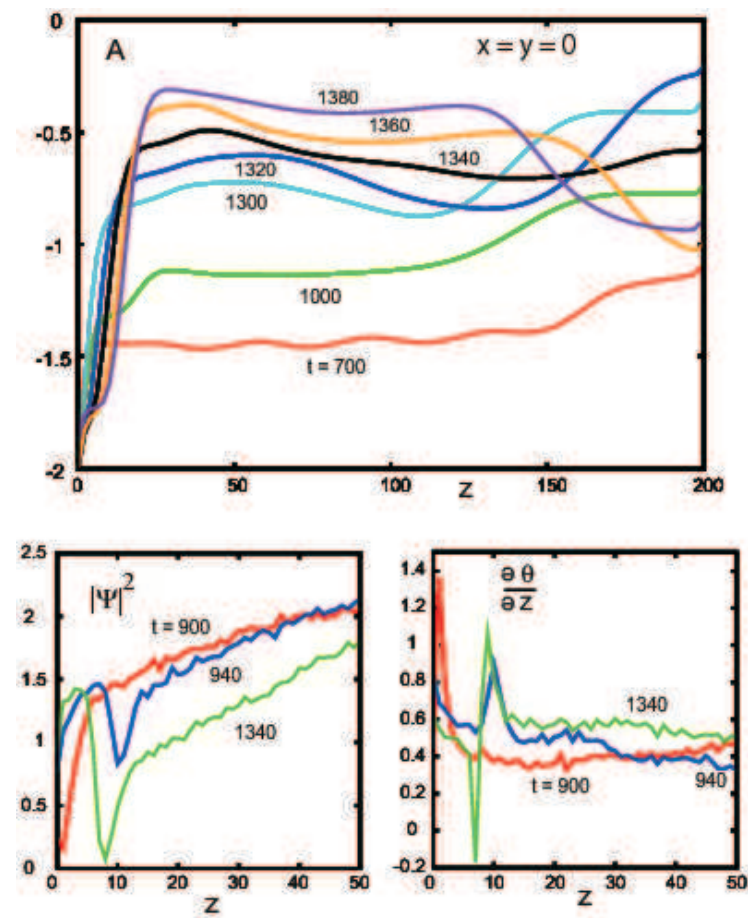

FIG. 1. (color online). Top: scaled temperature deviation $A(\boldsymbol{r}, t)$ at $x=y=0$ after application of heat flux from the top at $t=700,1000$, and $1300+20 n(0 \leq n \leq 4)$. The bulk region is gradually heated by repeated traversals of second sounds. Bottom: $|\Psi|^{2}\left(\propto \rho_{s}\right)$ (left) and $\partial \theta / \partial z\left(\propto v_{s z}\right)$ (right) at $t=900,940$, and 1340. A phase slip is being created at $z \sim 10$ in the latter two times (see the plates of Video 1).

where $\boldsymbol{v}_{n}$ is the normal fluid velocity and $\rho_{n}=\rho-\rho_{s} \cong \rho$. Then the normal fluid velocity $\boldsymbol{v}_{n}$ may be written as

$$
\boldsymbol{v}_{n}=-\rho^{-1} \boldsymbol{J}_{s} .
$$

Therefore $\boldsymbol{v}_{n}$ is much smaller than $\boldsymbol{v}_{s}=\rho_{s}^{-1} \mathbf{J}_{s}$ by the small factor $\rho_{s} / \rho$. This is justified for slow dynamic processes without first sounds near the lambda line.

In critical dynamics, the dynamic equations have been treated as Langevin equations with random source terms added ${ }^{4}$. In this work, we neglect the random source terms in Eqs.(17) and (18), which produce the thermal fluctuations of the order parameter $\psi$ and the entropy $s$. They may be neglected when the small-scale thermal fluctuations shorter than $\xi$ have been coarse-grained in the dynamic equations. The renormalization effect has already be accounted for in the renormalized coefficients.

\section{SIMULATION RESULTS}

In previous papers ${ }^{25,26}$, Eqs.(17) and (18) were integrated in one dimension, where phase slips play a major role in self-organized superfluid states, as in wire superconductors in electric field $27-30$. In this work, we present numerical results in three dimensions on a $200 \times 200 \times 200$ 

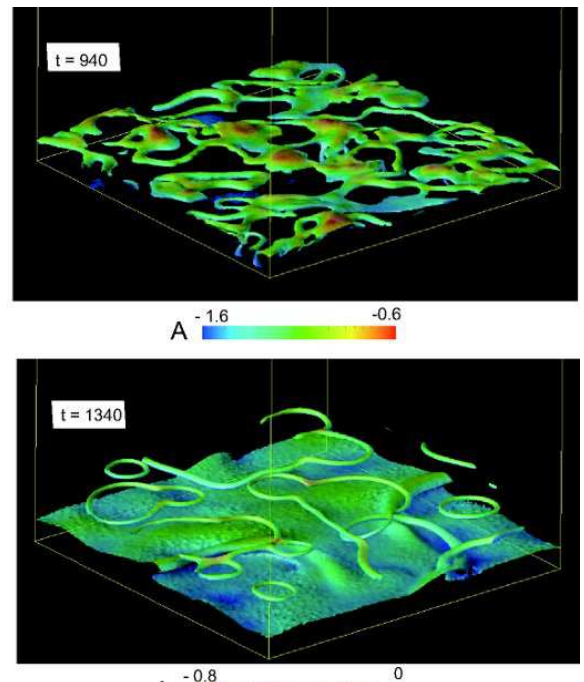

$A^{-0.8} \quad 0$

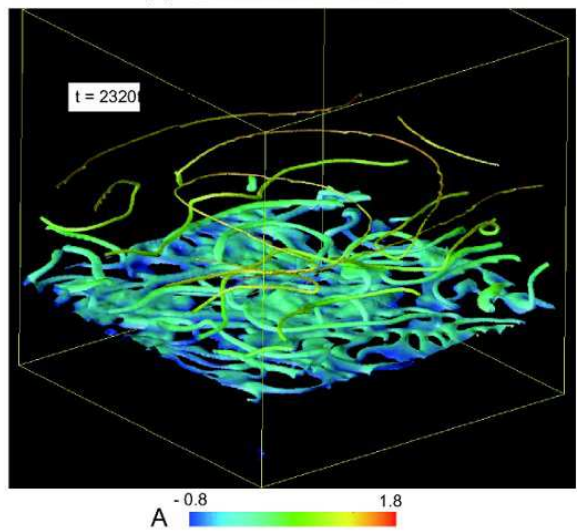

FIG. 2. Surfaces of $|\Psi|^{2}=0.8$ after application of heat flux from the top in a superfluid state in the same run as in Fig.1. Their colors represent the temperature deviation $A$ with the color map below the plates. Top: at $t=940$ a layer in the range $10 \lesssim z \lesssim 20$ is much disturbed, where a phase slip is going to appear. Temperature inhomogeneity is also apparent, for which see Fig.3. Middle: at $t=1340$ a sheetlike phase slip is created near the bottom. Vortices above the sheet are expanding. Bottom: at $t=2320$ the phase slip sheet is away from the bottom and is being broken into an aggregate of vortices. Video gives time evolution in the range $2000<t<3400$.

cubic lattice with the mesh size equal to $\tilde{\xi}$, so the corresponding cell length is $3.2 \mathrm{~mm}$. The time increment $\Delta t$ of integration is 0.01 in units of $\tilde{t}=0.5 \times 10^{-3} \mathrm{~s}$. The periodic boundary conditions are imposed in the $x y$ plane. At $z=0$ and 200 , we set $\Psi=0$ and assume homogeneity of the temperature and the heat flux in the $x y$ plane, supposing metallic plates. We prepared an equilibrium superfluid state with $A=-2$ for $t<0$. We then applied a constant heat flux at $z=h$, but we kept the bottom temperature at $A=-2(\tau=-2 \tilde{\tau})$ at $z=0$ for $t>0$. The scaled heat flux $\hat{Q}=\lambda^{*}(\partial A / \partial z)$ at the top is equal to 1 . This corresponds to a heat flux of $Q=11.2$, which is the value of $\lambda T_{\lambda} \tau / \xi$ at $\tau=\tilde{\tau}$. In the following we will explain our numerical results.

In Fig.1, we display profiles of the scaled reduced temperature $A$ vs $z$ (with $x=y=0$ ) at seven times $t=700,1000$, and $1260+40 n(0 \leq n \leq 4)$, where traversals of second sounds cause gradual heating of the bulk superfluid 17 . Since the bottom temperature is fixed, a transition layer supporting a temperature gradient grows near the bottom, where a heat flux of order 0.1 is from the liquid to the bottom plate. This heat output is $10 \%$ of the heat input from the top. In the lower plates, we show $|\Psi|^{2}$ and $\partial \theta / \partial z$ near the bottom at three times to illustrate formation of a phase slip, where $\theta$ is the phase of $\Psi=|\Psi| e^{i \theta}$. We confirmed that this phase slip formation in a narrow region occurs even if the mesh size is reduced to $0.2 \tilde{\xi}$. Here $\partial \theta / \partial z$ is equal to the superfluid velocity in the $z$ axis in units of $\hbar / m \bar{\xi}$, where $m$ is the ${ }^{4} \mathrm{He}$ mass. Note that the complex order parameter behaves as $\psi \propto e^{i k z}$ with $v_{s}=\hbar k / m$ in current-carrying superfluids and the bulk region is unstable for $k$ larger than $1 / \sqrt{3} \xi$ from linear stability analysis 34 . In our case, $\rho_{s}$ decreases towards zero and $v_{s}$ exceeds the critical value near the bottom, resulting in defect formation.

In Video 1, we display the surfaces of $|\Psi|^{2}=0.8$ near the bottom, which illustrates growth of vortices and phase slips. At $t=940$ disturbances of $\Psi$ are enhanced in a layer in the range $10 \lesssim z \lesssim 20$. See the lower plates in Fig.1. We can also see considerable inhomogeneity of the temperature. At $t=1340$, vortices are expanding and a sheetlike phase slip is at its birth. In our case under gravity, $|\Psi|^{2}$ decreases near the bottom. We observe that undulations on the sheet grow and detach to form vortices. At $t=2320$, the sheet is ramified into a vortex aggregate. Afterwards, sheetlike phase slips successively appear forming an expanding self-organized superfluid. As well as vortex proliferation, vortex aggregates sometimes fuse into sheetlike phase slips. These temporal changeovers occur on the system scale with large-amplitude second sounds. No clear boundary can be seen between the self-organized and ordinary superfluid regions, but high-density vortices are undergoing large-scale temporal fluctuations in the transition region.

In Fig.3, the inhomogeneity of $A(\boldsymbol{r}, t)$ is shown in the $x$-y plane at $z=20$ taken at $t=940$. It originates from the relation (16) for the entropy and the temperature. That is, if $\rho_{s}$ increases in some regions, the temperature there tends to increase if the entropy change is slower.

In Fig.4, time evolution of $A$ is shown at three heights with $x=y=0$ in a longer time range $10^{3}<t<3.4 \times 10^{3}$ in the same run producing Figs. 1 and 2. For $t<2000$ the three points are in an ordinary superfluid and are nearly uniformly heated. Subsequent zigzag temperature changes are due to the passage of platelike defect aggregates. A similar plot was given by Moeur et al $\underline{19}$, where the temperatures at three thermometers attached to the side wall became flat during the passage of a selforganized region. In our simulation, a train of defects eventually arrived at the top and a normal fluid with a steep temperature gradient emerged from the top. 


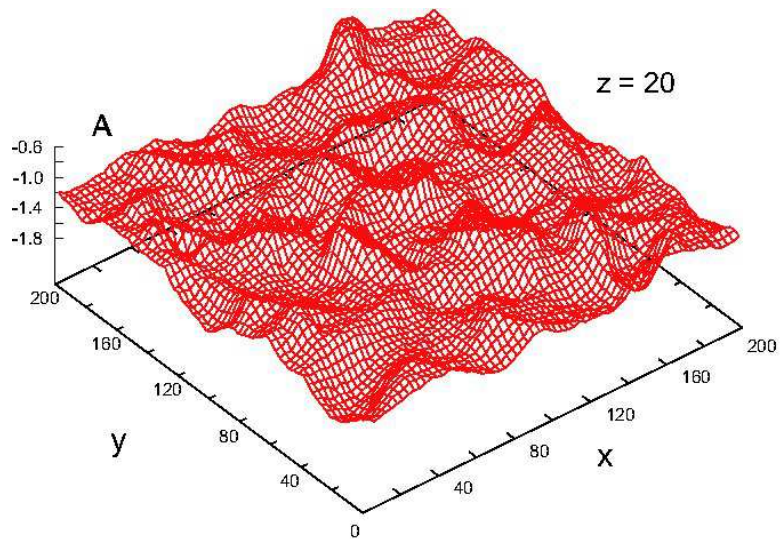

FIG. 3. (color online). Scaled temperature deviation $A(\boldsymbol{r}, t)$ in the $x$ - $y$ plane at $z=20$ taken at $t=940$ in the same run as in Fig.1 and Video 1, corresponding to the top plate in Video 1. A phase slip is being created with considerable temperature inhomogeneity, where $\langle A\rangle=-1.22$ and $\left\langle(A-\langle A\rangle)^{2}\right\rangle=0.156^{2}$ with $\langle\cdots\rangle$ denoting the areal average.

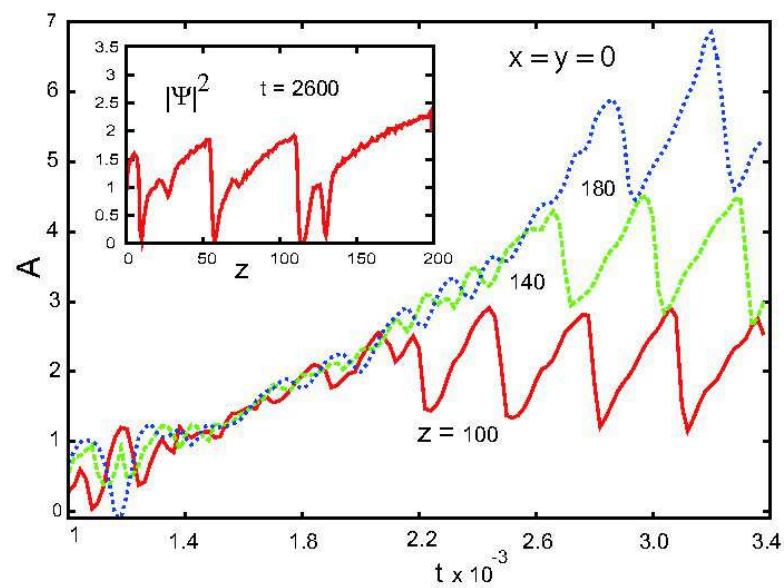

FIG. 4. (color online). Scaled temperature deviation $A(\boldsymbol{r}, t)$ at $z=100,140$, and 180 with $x=y=0$ after application of heat flux from the top in the time range $1000<t<3400$ in the same run as in Figs. 1 and 2. Inset: $|\Psi|^{2}$ vs $z$ at $t=2600$, which is zero at defect points. At this time, self-organized and ordinary superfluid regions are separated at $z \sim 130$.

In Fig.4, the advancing speed of the self-organized region is about 0.1 (in units of $\tilde{\xi} / \tilde{t}$ ) or $3 \mathrm{~cm} / \mathrm{s}$, while it was $2 \times 10^{-3} \mathrm{~cm} / \mathrm{s}$ in one of the experimental data (see Fig. 2 of Ref.19). Note that the speed of this phase change is determined by the rate of heat input to the system. To check this aspect, we fixed the bottom heat flux at the top value 1 and resumed the integration using the data at $t=2500$ in the run producing Figs.1-4. Then we could realize a dynamical steady state with a self-organized superfluid in a lower part and an ordinary superfluid in an upper part. Hereafter the times are 2500 plus those after the restart.
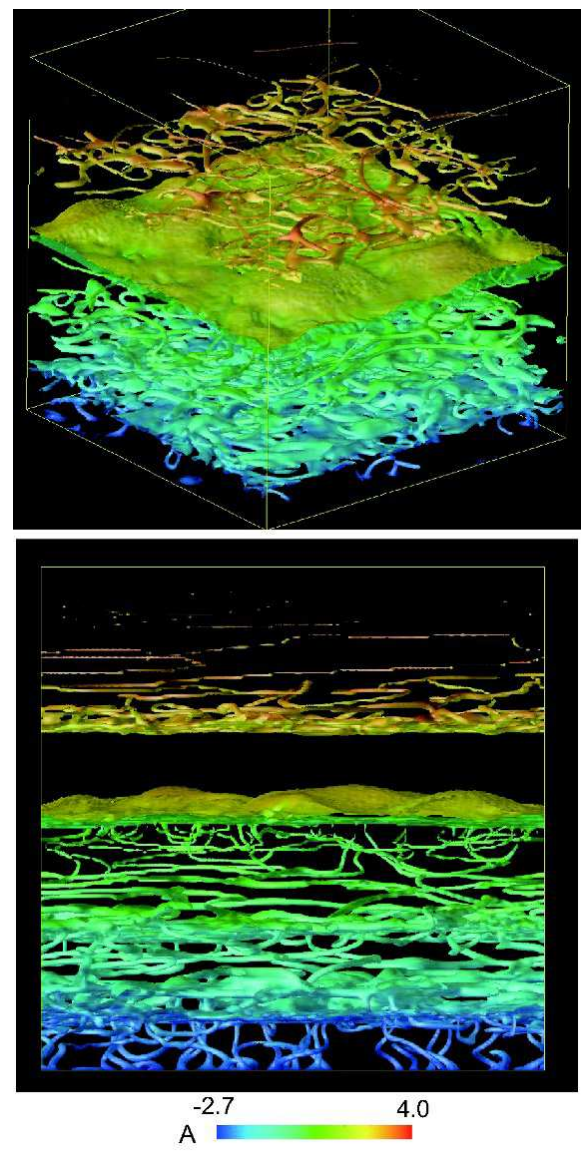

FIG. 5. Surfaces of $|\Psi|^{2}=1.2$ viewed from two angles in a dynamical steady state at $t=3840$. Their colors represent the temperature deviation $A$ with the color map below the plates. A sheetlike phase slip can be seen at the middle, while the other defects are aggregates of vortices.

In Fig.5, we display snapshots of the surfaces of $|\Psi|^{2}=$ 1.2 at $t=3840$ from above and side. The defect structure here is composed of a single sheetlike phase slip in the middle and platelike vortex aggregates. The vortices are highly connected one another forming a network. Some vortices start from the phase slip sheet or from the bottom wall. In this configuration, defects are absent in a layer region above the phase slip plane, but above this layer a vortex aggregate appears to form an interface between the self-organized and ordinary superfluid regions.

In Fig.6, we present the scaled temperature deviation $A(\boldsymbol{r}, t)$ vs $z$ with $x=y=0$ at three consecutive times in the dynamical steady state. It shows considerable displacive motions of the defect structure on a time scale of 70. The temperature variations are stepwise at a phase slip and are more gradual across vortex aggregates. In accord with the balance relation (5), the zig-zag curves of $A$ are separated from the line of $\tau=0$ by 2.5 , which is our numerical result of $\tau_{\text {sos }} / \tilde{\tau}$. In agreement with this result, the experimental formula (6) yields

$$
\tau_{\mathrm{sos}} / \tilde{\tau}=11.2^{0.813} / 2.5=2.85
$$




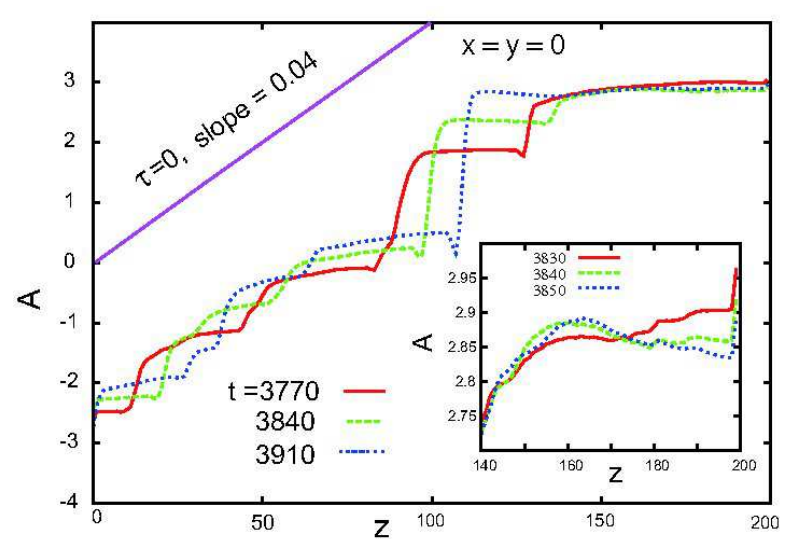

FIG. 6. (color online). Scaled temperature deviation $A(\boldsymbol{r}, t)$ vs $z(x=y=0)$ at $t=3770,3840$, and 3910 in a dynamical steady state in the same run as in Fig.4. Heat fluxes at the top and bottom are fixed at a common value. Significant defect movements can be seen. Line of $\tau=0$ is also shown. Inset: $A$ vs $z$ at $t=3830,3840$, and 3850 in the ordinary superfluid region, indicating standing second sound waves.

Note that phase slips in the one-dimensional simulation ${ }^{25}$ already gave $\tau_{\text {sos }}$ in agreement with the experiment $\underline{19}$. In the inset, we demonstrate the presence of second sounds oscillating on a time scale of 10 (smaller than those in Fig.1) in the upper ordinary superfluid region.

In Fig.7, we show the phase $\theta$ and the gradient amplitude $|\nabla \Psi|^{2}$ in the $x-z$ plane at $y=0$ using the same data as in Fig.5 at $t=3840$. These are cross-sectional profiles illustrating the complex defect structure. Around the defects, $\theta$ varies steeply in the upper plate, while peaks and ridges appear in the lower plate. Here we have $|\nabla \Psi|^{2} \cong|\Psi|^{2}|\nabla \theta|^{2} \propto \rho_{s} v_{s}^{2}$ around the defects.

\section{SUMMARY AND REMARKS}

In summary, we have investigated the self-organized superfluid state on earth under a fixed downward heat flux $Q=11.2 \mathrm{erg} / \mathrm{cm}^{2}$ s from the top in a cubic cell of 3.2 $\mathrm{mm}$ length. The periodic boundary condition has been imposed in the horizontal directions.

First, at a fixed bottom temperature, we have examined how vortices and phase slips appear near the bottom and how a self-organized region expands upward. Second, by setting the bottom heat flux equal to the top heat flux, we have realized a dynamical steady state in which the self-organized and ordinary superfluids coexist. The space-time fluctuations are highly enhanced, where a sheetlike phase slip often changes into a vortex aggregate and vice verse. In fact, in Fig.5, we can see only a single sheetlike phase slip, while numerous vortex lines form platelike networks. Our numerical value of $\tau$ in Eq.(2) (the distance from the lambda line) in selforganized superfluid states is in good agreement with the
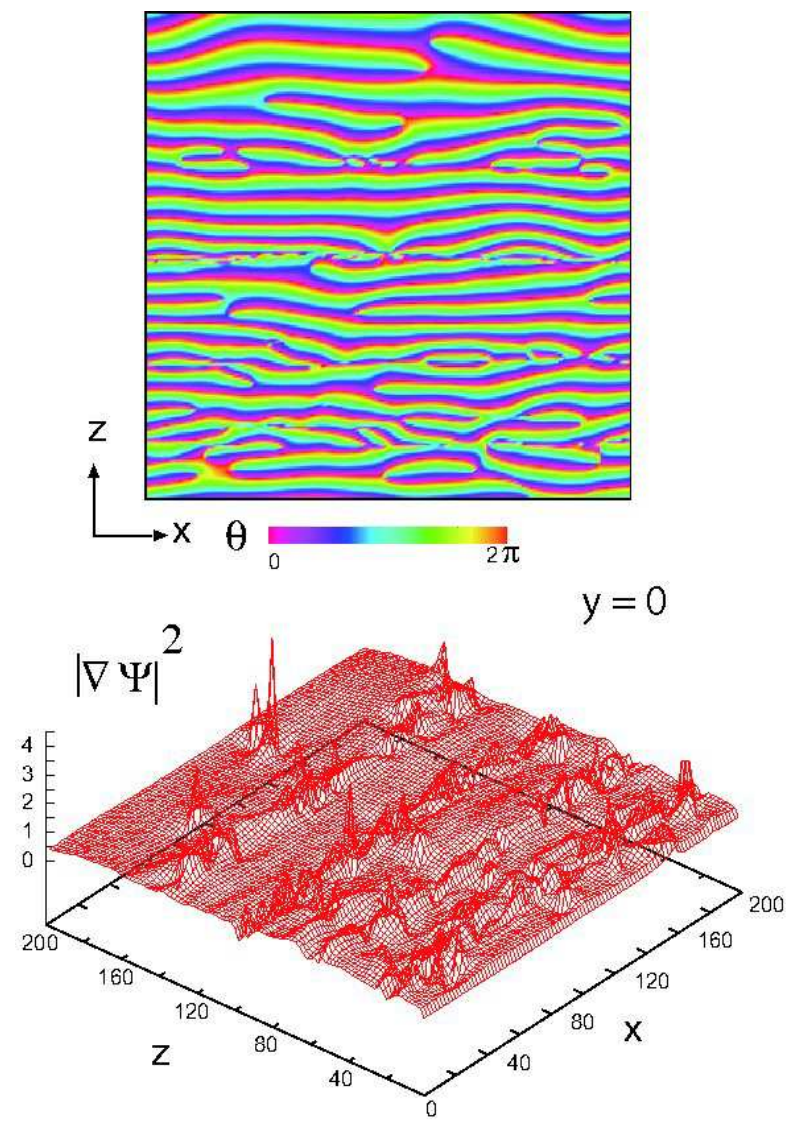

FIG. 7. (color online). Phase $\theta(\boldsymbol{r}, t)$ of $\Phi(\boldsymbol{r}, t)$ (upper plate) in the range $0 \leq \theta<2 \pi$ according to the color bar and $|\nabla \Phi|^{2}$ (lower plate) in the $x$ - $z$ plane at $y=0$ taken at $t=3840$, where use is made of the same data as in Fig.5.

experimental $\tau_{\text {sos }}$ in Eq.(6).

We estimate the temperature drop at a phase slip as

$$
(\Delta T)_{p}=Q \ell_{\mathrm{p}} / \lambda\left(\tau_{\mathrm{sos}}\right),
$$

where $\ell_{\mathrm{p}}$ is the thickness of a phase slip $(\sim \xi)$ and $\lambda\left(\tau_{\text {sos }}\right)$ is the thermal conductivity in Eq. (9) at $\tau=\tau_{\text {sos. }}$. If the defects consist of phase slips only, their density $n_{\mathrm{p}}\left(<\ell_{\mathrm{p}}^{-1}\right)$ per unit length satisfies $n_{\mathrm{p}}(\Delta T)_{\mathrm{p}}=T_{\lambda} G$ in self-organized superfluid states. Use of Eqs.(9), (12), and (24) yields

$$
n_{\mathrm{p}} \ell_{\mathrm{p}}=G T_{\lambda} \lambda\left(\tau_{\mathrm{sos}}\right) / Q=\left(\tau_{\mathrm{son}} / \tau_{\mathrm{sos}}\right)^{x_{\lambda}} .
$$

This estimation is consistent with the results in the onedimensional simulations 25,26 . Here the required inequality $n_{\mathrm{p}}<\ell_{\mathrm{p}}^{-1}$ holds for $Q>1$. In accord with this, selforganized superfluid states were observed only for $Q>1$ in the experiment $\frac{19}{}$. Notice that the vortex aggregates in Fig.5 also give rise to temperature drops comparable to that of a phase slip as in Fig.6.

A self-organized superfluid expands upward if the heat input from the top is larger than the heat output from the bottom. After its arrival at the top, a normal fluid begins to expand downward (not shown in this paper). There- 
fore, there can also be coexistence of a self-organized superfluid and a normal fluid. This coexistence was realized in our previous simulation 25 by cooling a normal fluid at the bottom below the transition. In such cooling, a self-organized superfluid region grows upward to reach the top and then an ordinary superfluid region appears to expand downward. On heating or cooling from a boundary wall without gravity or at large $Q$, a HeI-HeII interface appears and its movement leads to the phase transformation between normal fluid and superfluid.

Phase slips are well-known singular entities observed in quasi-one-dimensional superconductors ${ }^{30}$ and ${ }^{4} \mathrm{He}^{31}$. However, under an external potential, they can emerge as sheets in three-dimensional geometries. The role of such a potential becomes relevant on approaching the criticality. In the case of gravity, this crossover occurs extremely close to the lambda line. Self-organized superfluidity may be realized more easily in an inhomogeneous, intense electric or magnetic field, as stated in Sec.I, or under high-speed rotation.

In the literature ${ }^{14-16}$, vortex generation after quenching of ${ }^{4} \mathrm{He}$ was proposed but it has not been detected in subsequent experiments, where crossing of the lambda line by a mechanical expansion is supposed. A similar quenching method (using the piston effect) was used to induce phase separation in fluids near the gas-liquid critical point ${ }^{35}$. In a decompression experiment near the superfluid transition, however, we should examine how the phase transition is induced by sounds. As recently reported ${ }^{36}$, first sounds emitted from a boundary wall propagate throughout the cell with a stepwise pressure decrease at the wave front. They are multiply reflected at the boundary walls until dissipated. If the sound speed is $10^{4} \mathrm{~cm} / \mathrm{s}$ and the cell length is $1 \mathrm{~cm}$, the acoustic traversal time is $10^{-4} \mathrm{~s}$ and is much longer than the relaxation time of the order parameter except extremely close to the lambda line. Second sounds are also emitted from the boundary walls with appearance of superfluid. Further investigation of these processes is thus needed.

\section{ACKNOWLEDGMENTS}

One of the authors (A.O.) thanks R. Duncan for stimulating discussions in an early stage of this work. This work was supported by Grants-in-Aid for the Global COE program "The Next Generation of Physics, Spun from Universality and Emergence" of Kyoto University from the Ministry of Education, Culture, Sports, Science and Technology of Japan.
1 G. Ahlers, in The Physics of Liquid and Solid Helium, Part I, K.H. Bennemann and J.B. Ketterson, eds. (Wiley, New York, 1976), p.85; Phys. Rev. A 8, 530 (1973).

2 G. Ahlers, Rev. Mod. Phys. 52, 489 (1980); J. A. Lipa, D. R. Swanson, J. A. Nissen, T. C. P. Chui, and U. E. Israelsson, Phys. Rev. Lett. 76, 944 (1996).

3 W.Y. Tam and G. Ahlers, Phys. Rev. B 32, 5932 (1985); M. Dingus, F. Zhong and H. Meyer, J. Low Temp. Phys. 65, 185 (1986).

4 P.C. Hohenberg and B.I. Halperin, Rev. Mod. Phys. 49, 435 (1977).

5 A. Onuki, Phase Transition Dynamics (Cambridge University Press, Cambridge, 2002). See Fig.2.17 for a gravityinduced interface and Fig.6.25 for a heat-induced interface in this book.

6 P.B. Weichman, A.W. Harter, and D.L. Goodstein, Rev. Mod. Phys. 73, 1 (2001).

7 D. Erben and F. Pobell, Phys. Lett. 26 A, 368 (1968); P. Leiderer and F. Pobell, Z. Phys. 223, 378 (1969).

8 M. Bhagat and R.A. Rasken, Phys. Rev. A 3, 340 (1971); 4, 264 (1971); 5, 2297 (1972).

9 R.V. Duncan, G. Ahlers and V. Steinberg, Phys. Rev. Lett. 60, 1522 (1988).

10 A. W. Harter, R. A. M. Lee, A. Chatto, X. Wu, T. C. P. Chui, and D. L. Goodstein, 84, 2195 (2000).

11 R. Nishida, J. Taniguchi, R. Nomura, and Y. Okuda, J. Low Temp. Phys. 148, 109 (2007). Here a HeI-HeII interface was visualized for $Q \sim 4 \times 10^{4}$.

12 A. Onuki, J. Low Temp. Phys. 50, 433 (1983); ibid. 55, 309 (1984); A. Onuki and Y. Yamazaki, ibid. 103, 131 (1996).

13 R. Haussmann and V. Dohm Phys. Rev. Lett. 67, 3404 (1991); Phys. Rev. B 46, 6361 (1992); Z. Phys. B 87, 229
(1992); R. Haussmann, Phys. Rev. B 60, 12349 (1999).

14 W. H. Zurek, Phys. Rep. 276, (1996).

15 J. Rivers, ibid. 84, 1248 (2000); R. Monaco, J. Mygind, and R. J. Rivers, Phys. Rev. Lett. 89, 080603 (2002).

16 M. E. Dodd, P. C. Hendry, N. S. Lawson, P. V. E. McClintock, and C. D. H. Williams, Phys. Rev. Lett. 81, 3703 (1998); V. B. Efimov, O. J. Griffiths, P. C. Hendry, G. V. Kolmakov, P. V. E. McClintock, and L. Skrbek, Phys. Rev. E 74, 056305 (2006).

17 G. Ahlers, Phys. Rev. 171, 275 (1968).

18 V.L. Ginzburg and A.A. Sobaynin, J. Low Temp. Phys. 49, 507 (1982).

19 W.A. Moeur, P.K. Day, F-C Liu, S.T.P. Boyd, M.J. Adriaans and R.V. Duncan, Phys. Rev. Lett. 78, 2421 (1997).

20 D.A. Sergatskov, A.V. Babkin, S.T.P. Boyd, R.A.M. Lee, and R.V. Duncan, J. Low Temp. Phys. 134, 517 (2004).

21 A.R. Chatto, R.A.M. Lee, R.V. Duncan, and D.L. Goodstein, J. Low Temp. Phys. 148, 519 (2007).

22 J. Machta, D. Candela, and R.B. Hallock Phys. Rev. E 47, 4581 (1993).

23 R. J. Donnelly, Quantized Vortices in He II, (Cambridge University Press, Cambridge, 1991).

24 G. Ahlers, Phys. Rev. Lett. 22, 54 (1969).

25 A. Onuki, J. Low Temp. Phys. 104, 133 (1996). Here a train of phase slips gave $\tau_{\text {sos }}=2.5 \times 10^{-8}$ for $Q=3.4$ in agreement with the experimetal formula $(6)^{19}$.

26 P.B. Weichman and J. Miller, J. Low Temp. Phys. 119, $155(2000)$.

27 J.S. Langer and V. Ambegaokar, Phys. Rev. 164, 498 (1967); D. E. McCumber and B. I. Halperin, Phys. Rev. B 1, 1054 (1970).

28 L. Kramer and Baratoff, Phys. Rev. Lett., 38, 518 (1977). 
29 B. I. Ivlev and N. B. Kopnin, J. Low Temp. Phys. 44, 453 (1981).

30 W. J. Skocpol, M. R. Beasley, and M. Tinkham, J. Low Temp. Phys. 16, 145 (1974); W. J. Skocpol and M. Tinkham, Rep. Prog. Phys. 1975 38, 1049 (1975); M. Tian, J. Wang, J. S. Kurtz, Y. Liu, M. H. W. Chan, T. S. Mayer, and T. E. Mallouk, Phys. Rev. B 71, 104521 (2005).

31 O. Avenel and E. Varoquaux, Phys. Rev. Lett. 55, 2704 (1985); Y. Sato, A. Joshi, and R. Packard, Phys. Rev. B, 76, 052505 (2007).

32 A. Onuki, Jpn. J. Appl. Phys. 26, 365 (1987). Here some discussions were also given on current-induced interfaces in ${ }^{3} \mathrm{He}-{ }^{4} \mathrm{He}$ mixtures

33 L.P. Pitaevskii, Zh. Eksp. Teor. Fiz. 35, 408 (1958) [Sov. Phys. JETP. 8, 282 (1959)].

34 H.J. Mikeska, Phys.Rev. 179, 166 (1969); L. Kramer, Phys. Rev. 179, 149 (1969).

${ }^{35}$ F. Perrot, D. Beysens, Y. Garrabos, T. Frohlich, P. Guenon, M. Bonetti and P. Bravais, Phys. Rev. E 59, 3079 (1999).

36 Y. Miura, S. Yoshihara, M. Ohnishi, K. Honda, M. Matsumoto, J. Kawai, M. Ishikawa, H. Kobayashi, and A. Onuki, Phys. Rev. E 74, 010101 (R) (2006); A. Onuki, Phys. Rev. E 76, 061126 (2007). 